\title{
Theoretical Approach to the Energy Resolution of a Scintillation Spectrometer with Several Photodetectors
}

\author{
Victor V. Samedov \\ National Research Nuclear University MEPhI (Moscow Engineering Physics Institute) \\ v-samedov@yandex.ru
}

\begin{abstract}
The exact mathematical description of processes at registration of incident particles by a scintillation spectrometer with several photodetectors allows receiving correct formulae for the mean value and the variance of the amplitude at the output of photodetectors, and for the covariance between signals of photodetectors. It was shown that using the relative covariance of signals allows the experimental determination of the covariance in the light yield and the Fano factor in a scintillator. The important advantage of the proposed method is its independence on the gains and the noises of photodetectors electronics.
\end{abstract}

Index Terms - scintillation spectrometer, scintillation detector, photodetector, energy resolution, covariance between signals, Fano factor, light yield.

\section{INTRODUCTION}

$\mathrm{T}$ HERE are a number of processes in scintillation detectors that lead to output signal broadening in response to impinging monochromatic radiation. There are semiempirical formulae that account for the contribution of different factors to the energy resolution of scintillation detectors [1], [2], [3]. However, these formulae do not contain any information on relationship of these contributions with scintillator characteristics and characteristics of the processes that occur in a scintillation detector at registration of incident particles.

The correct formula for the variance of the output signal of a scintillation detector cannot be semiempirical. It must result strictly from the exact mathematical description of the processes that take place at registration of the incident particle by a scintillation detector. Only such formula has the predictive ability. Only in this case dependence of the energy resolution on characteristics of scintillation crystal and other parameters of the detector can be revealed, and the conditions at which the characteristics of the processes can be derived from the output signal. As the process of signal formation at the output of detector represents a random branching cascade process, the formalism of probability generating functions (PGF) is the most adequate for its formulation [4].

Our approach to formulation of the mathematical model of an incident particle registration by a scintillation detector is based on the approach developed in formulation of the

Manuscript received May 29, 2019

Victor V. Samedov is with the National Research Nuclear University MEPhI (Moscow Engineering Physics Institute), 31, Kashirskoye Shosse, 115409, Moscow, Russian Federation (telephone: 007-495-323-9038, email: v-samedov@yandex.ru) theory of shower spectrometers in [5], and published in [6] and [7].

\section{MATHEMATICAL MODEL OF A SCINTILLATION DETECTOR}

Let us formulate the mathematical model of an incident particle registration by a scintillation detector with $N$ photodetectors, which we number with the index $n$, $(n=\overline{1, N})$. The process of an incident particle energy transformation into the output signals includes the following successive stages.

1. The stage of interaction of an incident particle with a scintillation crystal. Let divide the coordinate space into volume elements $\Delta V_{i},(i=\overline{1, I})$ that belong to the detector volume $V$, and $\Delta V_{0}$ the exterior to the detector volume. An incident particle at interacting with the detector volume produces in the volume elements $\Delta V_{i}$ secondary particles of the type $\alpha,(\alpha=\overline{1, A})$ where index $\alpha$ determines secondary particles such as photons, electrons, positrons, phonons, and may include protons, if incident particles are protons or neutrons, and alpha-particles, if incident particles are alpha-particles. Let us divide the range of possible secondary-particle energies into the energy intervals $\Delta E_{j},(j=\overline{1, J})$ and the space of possible directions of secondary particles into solid-angle elements $\Delta \Omega_{k}, \quad(k=\overline{1, K})$. Let introduce a $A \times I \times J \times K$ dimensional random vector $\left\{N_{\text {aijk }}\right\}$, whose components $N_{\alpha i j k}$ are the number of secondary particles of the type $\alpha$ in the element of the phase space $\Delta \Gamma_{i j k}=\Delta V_{i} \Delta E_{j} \Delta \Omega_{k}$. Let introduce the probabilities $p_{\text {aijk }}^{c}$ of all possible combinations of components of the random vector $\left\{N_{\text {aijk }}^{c}\right\}$, which characterize all possible distributions of secondary particles in the phase space elements after finishing all processes of downconversion of the energy of the incident particle into the energy of secondary particles that cannot produce electron-hole pairs in the scintillation crystal.

2. The stage of electron-hole pairs generation. The scintillation properties are strongly related to the crystal structure. The scintillation crystals have the property of semiconductor material, and generation of light photons is 
going via the generation of electron-hole pairs. Let $f_{v_{\alpha i j k}}[s]$ be the PGF of the process of electron-hole pairs generation by a secondary particle of the type $\alpha$ from the element of the phase space $\Delta \Gamma_{i j k}$. Hereafter $s$ is the auxiliary variable of the probability generating function. In a semiconductor crystal, only secondary particles with energies greater than the threshold energy $E_{\alpha i j_{\min } k}$, which may depend on the point in the detector volume, and on the energy and moving direction of the secondary particle, can produce electronhole pairs. This fact can be accounted for by assuming that the mean value $\left\langle v_{\text {oijk }}\right\rangle$ and the variance $\sigma_{v_{\alpha i j k}}^{2}$ of the number of electron-hole pairs are equal to zero for secondary particles with energies less than the threshold energy $j \leq j_{\alpha \min }$.

Despite the diversity of mechanisms in various inorganic scintillators, the process of light photon emission in general consists of the following successive stages.

3. The stage of electron-hole pairs recombination. This stage is characterized by the probability of an electron-hole pair generated in the volume element $\Delta V_{i}$ to survive in recombination or to form an exciton $\varepsilon_{r \text { aijk }}$. This probability strongly depends on the stopping power of the secondary particle of the type $\alpha$ from the element of the phase space $\Delta \Gamma_{i j k}$. This stage is binomial with the PGF

$$
f_{r}[s]=1-\varepsilon_{r \alpha i j k}+\varepsilon_{r \alpha i j k} \cdot s,
$$

4. The stage of diffusion of carriers (electrons, holes and excitons) in a scintillator. This process is also binomial with the PGF

$$
f_{D}[s]=1-p_{D i i^{\prime}}+p_{D i i^{\prime}} \cdot s,(2)
$$

where $p_{D i i^{\prime}}$ is the probability of diffusion of an electronhole pair produced in the volume element $\Delta V_{i}$ to the volume element $\Delta V_{i^{\prime}},\left(i^{\prime}=\overline{1, I}\right)$.

5. The stage of luminescent centers activation. This stage obeys the binomial distribution with the PGF

$$
f_{a}[s]=1-\varepsilon_{a i^{\prime}}+\varepsilon_{a i^{\prime}} \cdot s,(3)
$$

where $\varepsilon_{a i^{\prime}}$ is the probability of luminescent center activation in the volume element $\Delta V_{i^{\prime}}$ by an electron-hole pair.

6. The stage of a light photon emission by the luminescent center. Because of a random spatial distribution of luminescent centers in the host matrix, the energy levels of each luminescent center experience different Stark shifts arising from the electric field of the surrounding ions. Therefore, the number of light photons per elementary mode of radiation is less than unity, and we can consider that photons are emitted by the luminescent centers independently. In this case, the process of a photon emission by the luminescent center belonging to the volume element $\Delta V_{i^{\prime}}$ is binomial with the PGF

$$
f_{e}[s]=1-\sum_{l=1}^{L} p_{l} \sum_{k^{\prime}=1}^{K^{\prime}} \varepsilon_{e i^{\prime} l k^{\prime}}+\sum_{l=1}^{L} p_{l} \sum_{k^{\prime}=1}^{K^{\prime}} \varepsilon_{e i^{\prime} l k^{\prime}} \cdot S,
$$

where $\varepsilon_{e i^{\prime} k^{\prime}}$ is the probability of the radiative transition with emission of a photon with the wavelength $\lambda_{l}$, $(l=\overline{1, L})$ and the direction in the solid-angle element $\Delta \Omega_{k^{\prime}},\left(k^{\prime}=\overline{1, K^{\prime}}\right)$. In (4) $p_{l}$ is the probability of the $l$ radiative transition mode $\left(\sum_{l=1}^{L} p_{l}=1\right)$.

It is necessary to notice that this model is also applicable to organic scintillators if the stage of electron-hole pairs generation is related to the stage of ionization and excitation of organic molecules; the stage of electron-hole pairs recombination to quenching processes; the stage of carriers diffusion to the process of excitation energy migration to other molecules; the stage of luminescent centers activation to the stage of internal conversion of the excitation energy into the energy of fluorescent transition; the stage of a light photon emission by the luminescent center to the stage of a fluorescent transition with emission of a light photon.

7. The stage of a photon collection on the entrance window of one of the photodetectors. The fundamental nature of indivisibility of a light photon requires that it must be counted by only one of photodetectors. Let $\tau_{n i^{\prime} l k^{\prime} m k^{\prime \prime}}$ be the probability of a photon with the wavelength $\lambda_{l}$ emitted in the solid-angle element $\Delta \Omega_{k^{\prime}}$ by the luminescent center in the $\Delta V_{i^{\prime}}$ to get the surface element $\Delta S_{n m}$, $(m=\overline{1, M})$ of the entrance window of $n$-th photodetector in the direction belonging to the solid-angle element $\Delta \Omega_{n k^{\prime \prime}},\left(k^{\prime \prime}=\overline{1, K^{\prime \prime}}\right)$ with respect to the surface normal. The PGF of this process has the form

$$
f_{\tau}\left[\left\{s_{n}\right\}\right]=1-\sum_{n=1}^{N} \sum_{m=1}^{M} \sum_{k^{\prime \prime}=1}^{K^{\prime \prime}} \tau_{n i^{\prime} l k^{\prime} m k^{\prime \prime}}+\sum_{n=1}^{N} \sum_{m=1}^{M} \sum_{k^{\prime \prime}=1}^{K^{\prime \prime}} \tau_{n i^{\prime} l k^{\prime} m k^{\prime \prime}} S_{n}
$$

where $\left\{s_{n}\right\}$ are the auxiliary variables of photodetectors.

8. The stage of a photon conversion to a photoelectron. The process of a photon conversion to a photoelectron in $n$ th photodetector is binomial with the PGF

$$
f_{\eta}[s]=1-\eta_{n l m k^{\prime \prime}}+\eta_{n l m k^{\prime \prime}} \cdot s,
$$

where $\eta_{n l m k}$ is the quantum efficiency of the surface element $\Delta S_{n m}$ of the $n$-th photodetector to the photon with the wavelength $\lambda_{l}$ crossing the entrance window in the direction belonging to the solid-angle element $\Delta \Omega_{n k^{\prime \prime}}$.

As the processes of successive stages $3-8$ are binomial, so the PGF of this compound cascade process is also binomial

$$
f_{\chi}\left[\left\{s_{n}\right\}\right]=1-\sum_{n=1}^{N} \chi_{n \alpha i j k}+\sum_{n=1}^{N} \chi_{n \alpha i j k} \cdot s_{n},
$$

where 
$\chi_{n \alpha i j k}=\varepsilon_{r \alpha i j k} \sum_{i^{\prime}=1}^{I} p_{D i i^{\prime}} \sum_{l=1}^{L} p_{l} \sum_{k^{\prime}=1}^{K^{\prime}} \sum_{m=1}^{M} \sum_{k^{\prime \prime}=1}^{K^{\prime \prime}} \varepsilon_{a i^{\prime}} \varepsilon_{e i^{\prime} l k^{\prime}} \tau_{n i^{\prime} l k^{\prime} m k^{\prime \prime}} \eta_{n l m k^{\prime \prime}}$

(8)

is the probability of a photoelectron creation in the $n$-th photodetector by a secondary particle of the type $\alpha$ from the element of the phase space $\Delta \Gamma_{i j k}$.

9. The stage of signal amplification in the $n$-th photodetector. Let $f_{\mathrm{g} n}\left[s_{n}\right],(n=\overline{1, N})$, be the PGF of the gain in the $n$-th photodetector with the mean value $\left\langle g_{n}\right\rangle$ and the variance $\sigma_{g n}^{2}$. Let $f_{\text {noise } n}\left[s_{n}\right]$ be the PGF of the electronic noise at the output of $n$-th photodetector with the zero mean value and the variance $\sigma_{\text {noise } n}^{2}$. 
For the model described above, the PGF of incident particles registration by a scintillation detector with several photodetectors is

$$
f_{\left\{Q_{n}\right\}}\left[\left\{s_{n}\right\}\right]=\lim _{I J K \rightarrow \infty} \sum_{c} p_{\alpha i j k}^{c} \prod_{\alpha=1}^{A} \prod_{i=1}^{I} \prod_{j=j_{\min }}^{J} \prod_{k=1}^{K}\left(f_{v_{\alpha i j k}}\left[1-\sum_{n=1}^{N} \chi_{n} \alpha i j k+\sum_{n=1}^{N} \chi_{n} \alpha_{i j k} f_{\mathrm{g} n}\left[s_{n}\right]\right]\right)^{N_{\alpha i j k}^{c}} \prod_{n=1}^{N} f_{\text {noise } n}\left[s_{n}\right]
$$

This PGF will be used for determination of the covariance of photodetectors signals.

If we set $\left\{s_{n}=s\right\},(n=\overline{1, N})$, then we have the PGF for the sum signal of scintillation spectrometer photodetectors

$$
f_{Q}[s]=\lim _{I J K \rightarrow \infty} \sum_{c} p_{\alpha i j k}^{c} \prod_{\alpha=1}^{A} \prod_{i=1}^{I} \prod_{j=j_{\min }}^{J} \prod_{k=1}^{K}\left(f_{v_{\alpha i j k}}\left[1-\sum_{n=1}^{N} \chi_{n \alpha i j k}+\sum_{n=1}^{N} \chi_{n \alpha i j k} f_{\mathrm{g} n}[s]\right]\right)^{N_{\alpha i j k}^{c}} \prod_{n=1}^{N} f_{\text {noise } n}[s],
$$

From the probability generating function (10), the mean value and the variance of the sum signal take forms:

$$
\begin{aligned}
& \langle Q\rangle=\left.\frac{\partial f_{Q}[s]}{\partial s}\right|_{s=1}=\lim _{I J K \rightarrow \infty} \sum_{c} p_{\alpha i j k}^{c} \sum_{\alpha=1}^{A} \sum_{i=1}^{I} \sum_{j=1}^{J} \sum_{k=1}^{K} N_{\alpha i j k}^{c}\left\langle v_{\alpha i j k}\right\rangle \sum_{n=1}^{N} \chi_{n \text { aijk }}\left\langle g_{n}\right\rangle, \text { (11) } \\
& \sigma_{Q}^{2}=\left.\frac{\partial f_{Q}^{2}[s]}{\partial s^{2}}\right|_{s=1}+\left.\frac{\partial f_{Q}[s]}{\partial s}\right|_{s=1}-\left(\left.\frac{\partial f_{Q}[s]}{\partial s}\right|_{s=1} ^{2}=\sigma_{\text {cov }}^{2}+\sigma_{\text {pair }}^{2}+\sigma_{\mathrm{ph}}^{2}+\sigma_{\text {gain }}^{2}+\sigma_{\text {noise }}^{2},\right.
\end{aligned}
$$

In (12),

$$
\begin{aligned}
& \sigma_{\mathrm{cov}}^{2}=\lim _{I J K \rightarrow \infty} \sum_{c} p_{\alpha i j k \alpha^{\prime} i^{\prime} j^{\prime} k^{\prime}}^{c} \sum_{\alpha=1}^{A} \sum_{i=1}^{I} \sum_{j=1}^{J} \sum_{k=1}^{K} N_{\alpha i j k}^{c}\left\langle v_{\alpha i j k}\right\rangle \sum_{n=1}^{N}\left\langle\chi_{n \alpha i j k}\right\rangle\left\langle g_{n}\right\rangle \sum_{\alpha^{\prime}=1}^{A} \sum_{i^{\prime}=1}^{I} \sum_{j^{\prime}=1}^{J} \sum_{k^{\prime}=1}^{K} N_{\alpha^{\prime} i^{\prime} j^{\prime} k^{\prime}}^{c}\left\langle v_{\alpha^{\prime} i^{\prime} j^{\prime} k^{\prime}}\right\rangle \sum_{n=1}^{N}\left\langle\chi_{n \alpha^{\prime} i^{\prime} j^{\prime} k^{\prime}}\right\rangle\left\langle g_{n}\right\rangle \\
& -\left(\lim _{I J K \rightarrow \infty} \sum_{c} p_{\alpha i j k}^{c} \sum_{\alpha=1}^{A} \sum_{i=1}^{I} \sum_{j=1}^{J} \sum_{k=1}^{K} N_{\alpha i j k}^{c}\left\langle v_{\alpha i j k}\right\rangle \sum_{n=1}^{N}\left\langle\chi_{n \alpha i j k}\right\rangle\left\langle g_{n}\right\rangle\right) \times \\
& \left(\lim _{I J K \rightarrow \infty} \sum_{c} p_{\alpha^{\prime} i^{\prime} j^{\prime} k^{\prime}}^{c} \sum_{\alpha^{\prime}=1}^{A} \sum_{i^{\prime}=1}^{I} \sum_{j^{\prime}=1}^{J} \sum_{k^{\prime}=1}^{K} N_{\alpha^{\prime} i^{\prime} j^{\prime} k^{\prime}}^{c}\left\langle v_{\alpha^{\prime} i^{\prime} j^{\prime} k^{\prime}}\right\rangle \sum_{n=1}^{N}\left\langle\chi_{n \alpha^{\prime} i^{\prime} j^{\prime} k^{\prime}}\right\rangle\left\langle g_{n}\right\rangle\right)
\end{aligned}
$$

is the variance of the output signal due to covariance of secondary particles in the phase space, where $p_{\alpha i j k \alpha^{\prime} i^{\prime} j^{\prime} k^{\prime}}^{c}$ is the joint distribution function of all possible combinations of secondary particles in the joint phase space.

$$
\begin{aligned}
& \sigma_{\text {pair }}^{2}=\lim _{I J K \rightarrow \infty} \sum_{c} p_{\alpha i j k}^{c} \sum_{\alpha=1}^{A} \sum_{i=1}^{I} \sum_{j=1}^{J} \sum_{k=1}^{K} N_{\alpha i j k}^{c} \sigma_{v_{\alpha i j k}}^{2}\left(\sum_{n=1}^{N}\left\langle\chi_{n \alpha i j k}\right\rangle\left\langle g_{n}\right\rangle\right)^{2} \\
& =\lim _{I J K \rightarrow \infty} \sum_{c} p_{\alpha i j k}^{c} \sum_{\alpha=1}^{A} \sum_{i=1}^{I} \sum_{j=1}^{J} \sum_{k=1}^{K} N_{\alpha i j k}^{c} F_{\alpha i j k}\left\langle v_{\alpha i j k}\right\rangle\left(\sum_{n=1}^{N}\left\langle\chi_{n \alpha i j k}\right\rangle\left\langle g_{n}\right\rangle\right)^{2}
\end{aligned}
$$

is the variance of the output signal due to fluctuations in the number of electron-hole pair. In (14), according to the Fano model [8], we assume that fluctuations in the number of electron-hole pairs are proportional to their average

$\sigma_{v_{\alpha i j k}}^{2}=F_{\alpha i j k}\left\langle v_{\alpha i j k}\right\rangle$,

where $F_{\alpha i j k}$ is the Fano factor.

$$
\sigma_{\mathrm{ph}}^{2}=\lim _{I J K \rightarrow \infty} \sum_{c} p_{\alpha i j k}^{c} \sum_{\alpha=1}^{A} \sum_{i=1}^{I} \sum_{j=1}^{J} \sum_{k=1}^{K} N_{\alpha i j k}^{c}\left\langle v_{\alpha i j k}\right\rangle\left(\sum_{n=1}^{N}\left\langle\chi_{n \alpha^{\prime} i^{\prime} j^{\prime} k^{\prime}}\right\rangle\left\langle g_{n}\right\rangle^{2}-\left(\sum_{n=1}^{N}\left\langle\chi_{n \alpha i j k}\right\rangle\left\langle g_{n}\right\rangle\right)^{2}\right)
$$

is the variance of the output signal due to fluctuations in the number of photoelectrons in photodetectors.

$$
\sigma_{\text {gain }}^{2}=\lim _{I J K \rightarrow \infty} \sum_{c} p_{\alpha i j k}^{c} \sum_{\alpha=1}^{A} \sum_{i=1}^{I} \sum_{j=1}^{J} \sum_{k=1}^{K} N_{\alpha i j k}^{c}\left\langle v_{\alpha i j k}\right\rangle \sum_{n=1}^{N}\left\langle\chi_{n \alpha^{\prime} i^{\prime} j^{\prime} k^{\prime}}\right\rangle \sigma_{g n}^{2}
$$

is the variance of the output signal due to fluctuations in the electronic gains of photodetectors.

$$
\sigma_{\text {noise }}^{2}=\sum_{n=1}^{N} \sigma_{\text {noise } n}^{2}
$$


is the variance due to electronic noise.

As the average number of electron-hole pairs is determined by the energy absorbed in the detector volume element, it is convenient to move from the particle distribution to the absorbed energy distribution as follows:

$N_{\text {aijk }}^{c}\left\langle v_{\text {aijk }}\right\rangle=W_{\text {aijk }}^{c} / \varepsilon_{\mathrm{e}-\mathrm{h} \text { aijk }}$,

where $W_{\text {aijk }}^{c}$ is the energy absorbed in the volume element $\Delta V_{i}$ given $N_{\text {aijk }}^{c}$ secondary particles of type $\alpha$ are in the phase space element $\Delta \Gamma_{i j k}$; and $\varepsilon_{\mathrm{e}-\mathrm{h} \alpha i j k}$ is the average energy of the electron-hole pair production.

Any detector is usually characterized by the energy resolution of the total absorption peak, when the total energy of the primary monoenergetic particle $E_{0}$ is absorbed in the detector volume

$$
\lim _{I J K \rightarrow \infty} \sum_{\alpha=1}^{A} \sum_{i=1}^{I} \sum_{j=1}^{J} \sum_{k=1}^{K} W_{\alpha i j k}^{c}=\sum_{\alpha=1}^{A} \iint_{V} \int_{E} d V d E d \vec{\Omega} \frac{\partial^{3} W_{\alpha}^{c}\left(E_{0}, \vec{r}, E, \vec{\Omega}\right)}{\partial V \partial E \partial \vec{\Omega}}=\sum_{\alpha=1}^{A} \int_{V} \int_{E} \int_{\Omega} d V d E d \vec{\Omega} w_{\alpha}^{c}\left(E_{0}, \vec{r}, E, \vec{\Omega}\right)=E_{0}
$$

where $w_{\alpha}^{c}\left(E_{0}, \vec{r}, E, \vec{\Omega}\right)$ is the differential density of absorbed energy in the detector volume given $N_{\alpha i j k}^{c}$ secondary particles of the type $\alpha$ are in the phase space element $\Delta \Gamma_{i j k}$.

For the case when primary monoenergetic particles are totally absorbed in the detector volume, the mean value of signal takes the form

$$
\langle Q\rangle=\sum_{\alpha=1}^{A} \int_{V} \int_{E} \int_{\Omega} d V d E d \vec{\Omega} u\left(E-E_{\alpha \min }(\vec{r}, E, \vec{\Omega})\right)\left\langle\frac{w_{\alpha}^{c}\left(E_{0}, \vec{r}, E, \vec{\Omega}\right)}{\varepsilon_{\mathrm{e}-\mathrm{h} \alpha}(\vec{r}, E, \vec{\Omega})}\left(\sum_{n=1}^{N}\left\langle\chi_{n \alpha}(\vec{r}, E, \vec{\Omega})\right\rangle_{D}\left\langle g_{n}\right\rangle\right)\right\rangle_{c}
$$

where the Heaviside unit function, $(u(x)=0$ for $x<0$ and $u(x)=1$ for $x \geq 0)$ accounts for the threshold energy of electron-hole pair production by secondary particles. The subscript $c$ of the angle brackets denotes averaging over all possible distributions of secondary particles in the phase space elements;

$$
\begin{aligned}
& \left\langle\chi_{n \alpha}(\vec{r}, E, \vec{\Omega})\right\rangle_{D}=\int_{V} d V^{\prime} \rho_{D}\left(\vec{r}, \vec{r}^{\prime}\right) \sum_{l=1}^{L} p_{l}\left(\lambda_{l}\right) \int_{\Omega^{\prime}} d \vec{\Omega}^{\prime} \varepsilon_{r \alpha}\left(\vec{r}, E, \vec{\Omega}, \vec{r}^{\prime}\right) \varepsilon_{a}\left(\vec{r}^{\prime}\right) \varepsilon_{e}\left(\vec{r}^{\prime}, \lambda_{l}, \vec{\Omega}^{\prime}\right) \times \\
& \int_{S_{n}} \int_{\widehat{\Omega}_{n}^{\prime \prime}} d S_{n} d \vec{\Omega}_{n}^{\prime \prime} \tau_{n}\left(\vec{r}^{\prime}, \lambda_{l}, \vec{\Omega}^{\prime}, S_{n}, \vec{\Omega}_{n}^{\prime \prime}\right) \eta_{n}\left(\lambda_{l}, S_{n}, \vec{\Omega}_{n}^{\prime \prime}\right)
\end{aligned}
$$

The subscript $D$ of the angle brackets denotes averaging over the diffusion probability density.

In a uniform scintillator the spreading of carriers produced at the point $\vec{r}$ is symmetrical about the origin, and the probability density of finding a carrier at the point $\vec{r}^{\prime}$ of the scintillator depends only on the relative distance between the points and obeys the normal distribution

$$
\rho_{D}\left(\vec{r}^{\prime}, \vec{r}\right)=\rho_{D}\left(\mid \vec{r}^{\prime}-\vec{r}\right)=\left(\sqrt{4 \pi} \lambda_{D}\right)^{-3} \exp \left(-\left(\vec{r}^{\prime}-\vec{r}\right)^{2} / 4 \lambda_{D}^{2}\right)
$$

with characteristic diffusion length

$$
\lambda_{D}=\sqrt{D \tau}
$$

where $D$ is the effective diffusion coefficient, and $\tau$ is the mean lifetime of carriers.

The Taylor expansion of the coefficient (22) up to the second order on displacement $\vec{r}^{\prime}-\vec{r}$ has the form

$$
\chi_{n \alpha}\left(\vec{r}^{\prime}, E, \vec{\Omega}\right)=\chi_{n \alpha}(\vec{r}, E, \vec{\Omega})+\left(\left(\vec{r}^{\prime}-\vec{r}\right) \cdot \nabla\right) \chi_{n \alpha}(\vec{r}, E, \vec{\Omega})+\frac{1}{2 !}\left(\left(\vec{r}^{\prime}-\vec{r}\right) \cdot \nabla\right)^{2} \chi_{n \alpha}(\vec{r}, E, \vec{\Omega}) .
$$

At averaging (25) over the probability density of diffusion (23), owing to isotropy the linear term vanishes, and the second order term comes to

$$
\begin{aligned}
& \left\langle\frac{1}{2 !}\left(\left(\vec{r}^{\prime}-\vec{r}\right) \cdot \nabla\right)^{2} \chi_{n \alpha}(\vec{r}, E, \vec{\Omega})\right\rangle_{D}=\frac{1}{2}\left\langle\left.\sum_{i, i^{\prime}=1}^{3}\left(\xi_{i}^{\prime}-\xi_{i}\right) \frac{\partial^{2} \chi_{n \alpha}(\vec{r}, E, \vec{\Omega})}{\partial \xi_{i} \partial \xi_{i}^{\prime}}\right|_{\xi_{i}, \xi^{\prime}=x, y, z}\left(\xi_{i^{\prime}}^{\prime}-\xi_{i^{\prime}}\right)\right\rangle_{D}= \\
& \left.\frac{1}{2} \sum_{i=1}^{3} \frac{\partial^{2} \chi_{n \alpha}(\vec{r}, E, \vec{\Omega})}{\partial \xi_{i}^{2}}\right|_{\xi_{i}=x, y, z}\left\langle\left(\xi_{i}^{\prime}-\xi_{i}\right)^{2}\right\rangle_{D}=\frac{1}{6} \Delta \chi_{n \alpha}(\vec{r}, E, \vec{\Omega})\left\langle\left(\vec{r}^{\prime}-\vec{r}\right)^{2}\right\rangle_{D}=\Delta \chi_{n \alpha}(\vec{r}, E, \vec{\Omega}) \lambda_{D}^{2}
\end{aligned},
$$

As a result, 


$$
\begin{aligned}
& \left\langle\chi_{n \alpha}(\vec{r}, E, \vec{\Omega})\right\rangle_{D}=\chi_{n \alpha}(\vec{r}, E, \vec{\Omega})+\Delta \chi_{n \alpha}(\vec{r}, E, \vec{\Omega}) \lambda_{D}^{2}=\left(1+\lambda_{D}^{2} \Delta\right) \chi_{n \alpha}(\vec{r}, E, \vec{\Omega})= \\
& =\sum_{l=1}^{L} p_{l}\left(\lambda_{l}\right)\left(1+\lambda_{D}^{2} \Delta\right) \int_{\Omega^{\prime}} d \vec{\Omega}^{\prime} \varepsilon_{r \alpha}(\vec{r}, E, \vec{\Omega}) \varepsilon_{a}(\vec{r}) \varepsilon_{e}\left(\vec{r}, \lambda_{l}, \vec{\Omega}^{\prime}\right) \int_{S_{n}} \int_{\Omega_{n}^{\prime \prime}} d S_{n} d \vec{\Omega}_{n}^{\prime \prime} \tau_{n}\left(\vec{r}, \lambda_{l}, \vec{\Omega}^{\prime}, S_{n}, \vec{\Omega}_{n}^{\prime \prime}\right) \eta_{n}\left(\lambda_{l}, S_{n}, \vec{\Omega}_{n}^{\prime \prime}\right)
\end{aligned}
$$

As we move to the absorbed energy distribution in the detector volume, the contributions to the output signal variance are $\sigma_{\mathrm{cov}}^{2}=\sum_{\alpha=1}^{A} \sum_{\alpha^{\prime}=1}^{A} \iint_{V} \int_{E} \int_{V^{\prime}} \int_{E^{\prime}} \int_{\Omega^{\prime}} d V d E d \vec{\Omega} d V^{\prime} d E^{\prime} d \vec{\Omega}^{\prime} u\left(E-E_{\alpha \min }(\vec{r}, E, \vec{\Omega})\right) u\left(E^{\prime}-E_{\alpha^{\prime} \min }\left(\vec{r}^{\prime}, E^{\prime}, \vec{\Omega}^{\prime}\right)\right) \times$

$\left\langle\frac{w_{\alpha}^{c}\left(E_{0}, \vec{r}, E, \vec{\Omega}\right)}{\varepsilon_{\mathrm{e}-\mathrm{h} \alpha}(\vec{r}, E, \vec{\Omega})}\left(\sum_{n=1}^{N}\left\langle\chi_{n \alpha}(\vec{r}, E, \vec{\Omega})\right\rangle_{D}\left\langle g_{n}\right\rangle\right) \frac{w_{\alpha^{\prime}}^{c}\left(E_{0}, \vec{r}^{\prime}, E^{\prime}, \vec{\Omega}^{\prime}\right)}{\varepsilon_{\mathrm{e}-\mathrm{h} \alpha^{\prime}}\left(\vec{r}^{\prime}, E^{\prime}, \vec{\Omega}^{\prime}\right)}\left(\sum_{n^{\prime}=1}^{N}\left\langle\chi_{n^{\prime} \alpha^{\prime}}\left(\vec{r}^{\prime}, E^{\prime}, \vec{\Omega}^{\prime}\right)\right\rangle_{D}\left\langle g_{n^{\prime}}\right\rangle\right)\right\rangle_{c}$,

$-\left(\sum_{\alpha=1}^{A} \iint_{V} \int_{\Omega} d V d E d \vec{\Omega} u\left(E-E_{\alpha \min }(\vec{r}, E, \vec{\Omega})\right)\left\langle\frac{w_{\alpha}^{c}\left(E_{0}, \vec{r}, E, \vec{\Omega}\right)}{\varepsilon_{\mathrm{e}-\mathrm{h} \alpha}(\vec{r}, E, \vec{\Omega})}\left(\sum_{n=1}^{N}\left\langle\chi_{n \alpha}(\vec{r}, E, \vec{\Omega})\right\rangle_{D}\left\langle g_{n}\right\rangle\right)\right\rangle_{c}\right)^{2}$

$\sigma_{\text {pair }}^{2}=\sum_{\alpha=1}^{A} \iint_{V} \int_{\Omega} d V d E d \vec{\Omega} u\left(E-E_{\alpha \min }(\vec{r}, E, \vec{\Omega})\right) \times$

$\left\langle F_{\alpha}(\vec{r}, E, \vec{\Omega}) \frac{w_{\alpha}^{c}\left(E_{0}, \vec{r}, E, \vec{\Omega}\right)}{\varepsilon_{\mathrm{e}-\mathrm{h} \alpha}(\vec{r}, E, \vec{\Omega})}\left(\sum_{n=1}^{N}\left\langle\chi_{n \alpha}(\vec{r}, E, \vec{\Omega})\right\rangle_{D}\left\langle g_{n}\right\rangle\right)^{2}\right\rangle_{c}$

$\sigma_{\mathrm{ph}}^{2}=\sum_{\alpha=1}^{A} \iint_{V} \int_{\Omega} d V d E d \vec{\Omega} u\left(E-E_{\alpha \min }(\vec{r}, E, \vec{\Omega})\right) \times$

$\left\langle\frac{w_{\alpha}^{c}\left(E_{0}, \vec{r}, E, \vec{\Omega}\right)}{\varepsilon_{\mathrm{e}-\mathrm{h} \alpha}(\vec{r}, E, \vec{\Omega})}\left(\sum_{n=1}^{N}\left\langle\chi_{n \alpha}(\vec{r}, E, \vec{\Omega})\right\rangle_{D}\left\langle g_{n}\right\rangle^{2}-\left(\sum_{n=1}^{N}\left\langle\chi_{n \alpha}(\vec{r}, E, \vec{\Omega})\right\rangle_{D}\left\langle g_{n}\right\rangle\right)^{2}\right)\right\rangle_{c}$

$\sigma_{\text {gain }}^{2}=\sum_{\alpha=1}^{A} \int_{V} \int_{E} \int_{\Omega} d V d E d \vec{\Omega} u\left(E-E_{\alpha \min }(\vec{r}, E, \vec{\Omega})\right)\left\langle\frac{w_{\alpha}^{c}\left(E_{0}, \vec{r}, E, \vec{\Omega}\right)}{\varepsilon_{\mathrm{e}-\mathrm{h} \alpha}(\vec{r}, E, \vec{\Omega})} \sum_{n=1}^{N}\left\langle\chi_{n \alpha}(\vec{r}, E, \vec{\Omega})\right\rangle_{D} \sigma_{g n}^{2}\right\rangle_{c}$

$\sigma_{\text {noise }}^{2}=\sum_{n=1}^{N} \sigma_{\text {noise } n}^{2}$

The expressions (21) (27) - (32) are the most general formulae for the average and the variance of a scintillation detector with several photodetectors and are the basis for various approximations. These expressions include the formulae for the average and the variance of any photodetector signal if we leave in the sum only one term for the given photodetector.

\section{APPROXIMATION FOR LOW-ENERGY X-RAYS}

For the following, we need approximation for low-energy X-rays, when we can ignore the process of multiple scattering of Xquantum and can consider that its energy is transmitted to the kinetic energy of a photoelectron at the point of interaction. If the volume of downconversion of the photoelectron energy into the energy of electron-hole pairs is small compared with the volume of the detector, we can consider that all electron-hole pairs are generated at the point of X-ray quantum interaction with the detector volume.

For uniform and isotropic scintillator with the only one radiative transition mode the expression (27) is simplified to

$$
\left\langle\chi_{n}(\vec{r}, E, \vec{\Omega})\right\rangle_{D}=S(E) Q T_{n}(\vec{r}),
$$

where $S(E)=\varepsilon_{r}(E) \varepsilon_{a}$ is the standard notation of the probability of the luminescent center activation by one electron-hole pair that can depend only on the electron energy; $Q$ is the standard notation of the quantum efficiency of the luminescence process defined as $Q / 4 \pi=\varepsilon_{e}\left(\vec{r}, \lambda_{l}, \vec{\Omega}^{\prime}\right)$;

$$
T_{n}(\vec{r})=\left(1+\lambda_{D}^{2} \Delta\right) \int_{\Omega^{\prime}} \frac{d \vec{\Omega}^{\prime}}{4 \pi} \int_{S_{n}} \int_{\vec{\Omega}_{n}^{\prime \prime}} d S_{n} d \vec{\Omega}_{n}^{\prime \prime} \tau_{n}\left(\vec{r}, \vec{\Omega}^{\prime}, S_{n}, \vec{\Omega}_{n}^{\prime \prime}\right) \eta_{n}\left(S_{n}, \vec{\Omega}_{n}^{\prime \prime}\right)
$$


is the probability of a photoelectron creation in the $n$-th photodetector by a photon emitted by the luminescent center at the point $\vec{r}$ of the scintillator.

In a uniform and isotropic scintillator $\varepsilon_{\mathrm{e}-\mathrm{h}}(\vec{r}, E, \vec{\Omega})=\varepsilon_{\mathrm{e}-\mathrm{h}}(E)$. For the case of the local absorption of the energy of an incident particle at the point $\vec{r}_{c}$ of the scintillator, the differential density of absorbed energy in the detector volume is

$$
w^{c}\left(E_{0}, \vec{r}, E\right)=\int_{\Omega} d \vec{\Omega} w^{c}\left(E_{0}, \vec{r}, E, \vec{\Omega}\right)=w^{c}\left(E_{0}, E\right) \delta\left(\vec{r}-\vec{r}_{c}\right) .
$$

The averaging over of all possible combinations of secondary particles in the phase space is factorized, and the averaging over the spatial coordinate is due to variation of the X-ray quantum interaction point.

In this case the mean value and the variance of the sum signal take forms

$$
\begin{aligned}
& \langle Q\rangle=\int_{E} d E u\left(E-E_{\min }\right)\left\langle\frac{w^{c}\left(E_{0}, E\right)}{\varepsilon_{\mathrm{e}-\mathrm{h}}(E)} S(E) Q\right\rangle_{c}\left\langle\sum_{n=1}^{N} T_{n}\left(\vec{r}_{c}\right)\left\langle g_{n}\right\rangle\right\rangle_{c}, \\
& \sigma_{\mathrm{cov}}^{2}=\int_{E} \int_{E^{\prime}} d E d E^{\prime} u\left(E-E_{\mathrm{min}}\right) u\left(E^{\prime}-E_{\mathrm{min}}\right)\left\langle\frac{w^{c}\left(E_{0}, E\right)}{\varepsilon_{\mathrm{e}-\mathrm{h}}(E)} S(E) Q \frac{w^{c}\left(E_{0}, E^{\prime}\right)}{\varepsilon_{\mathrm{e}-\mathrm{h}}\left(E^{\prime}\right)} S\left(E^{\prime}\right) Q\right\rangle\left\langle\left(\sum_{n=1}^{N} T_{n}\left(\vec{r}_{c}\right)\left\langle g_{n}\right\rangle\right)^{2}\right\rangle \\
& -\left(\int_{E} d E u\left(E-E_{\min }\right)\left\langle\frac{w^{c}\left(E_{0}, E\right)}{\varepsilon_{\mathrm{e}-\mathrm{h}}(E)} S(E) Q\right\rangle_{c}\left\langle\sum_{n=1}^{N} T_{n}\left(\vec{r}_{c}\right)\left\langle g_{n}\right\rangle\right\rangle_{c}\right)^{2} \\
& \sigma_{\text {pair }}^{2}=\int_{E} d E u\left(E-E_{\text {min }}\right)\left\langle F(E) \frac{w^{c}\left(E_{0}, E\right)}{\varepsilon_{\mathrm{e}-\mathrm{h}}(E)} S^{2}(E) Q^{2}\right\rangle_{c}\left\langle\left(\sum_{n=1}^{N} T_{n}\left(\vec{r}_{c}\right)\left\langle g_{n}\right\rangle\right)^{2}\right\rangle_{c}, \\
& \sigma_{\mathrm{ph}}^{2}=\int_{E} d E u\left(E-E_{\min }\right)\left\langle\frac{w^{c}\left(E_{0}, E\right)}{\varepsilon_{\mathrm{e}-\mathrm{h}}(E)} S(E) Q\right\rangle_{c}\left\langle\sum_{n=1}^{N} T_{n}\left(\vec{r}_{c}\right)\left\langle g_{n}\right\rangle^{2}\right\rangle_{c} \\
& -\int_{E} d E u\left(E-E_{\min }\right)\left\langle\frac{w^{c}\left(E_{0}, E\right)}{\varepsilon_{\mathrm{e}-\mathrm{h}}(E)} S^{2}(E) Q^{2}\right\rangle_{c}\left\langle\left(\sum_{n=1}^{N} T_{n}\left(\vec{r}_{c}\right)\left\langle g_{n}\right\rangle\right)^{2}\right\rangle_{c} \\
& \sigma_{\text {gain }}^{2}=\int_{E} d E u\left(E-E_{\text {min }}\right)\left\langle\frac{w^{c}\left(E_{0}, E\right)}{\varepsilon_{\mathrm{e}-\mathrm{h}}(E)} S(E) Q\right\rangle_{c}\left\langle\sum_{n=1}^{N} T_{n}\left(\vec{r}_{c}\right) \sigma_{g n}^{2}\right\rangle_{c},
\end{aligned}
$$

For the scintillation spectrometer with the one photodetector, for comparing with existing in literature formulae, it is more convenient to use the relative variance related to the energy resolution of the spectrometer by the expression

$\frac{\Delta E}{E}=2.36 \eta_{Q}$,

where $\Delta E$ is the full width at half maximum (FWHM) of the line with the energy $E$.

The relative variance for a scintillation spectrometer with the one photodetector is

$$
\eta_{Q}^{2}=\frac{\sigma_{Q}^{2}\left(E_{0}\right)}{\left\langle Q\left(E_{0}\right)\right\rangle^{2}}=\eta_{\text {cov }}^{2}+\eta_{\text {pair }}^{2}+\eta_{p h}^{2}+\eta_{\text {gain }}^{2}+\eta_{\text {noise }}^{2}
$$

where

$$
\begin{aligned}
& \left\langle Q\left(E_{0}\right)\right\rangle=\int_{E} d E u\left(E-E_{\min }\right)\left\langle\frac{w^{c}\left(E_{0}, E\right)}{\varepsilon_{\mathrm{e}-\mathrm{h}}(E)} S(E) Q\right\rangle_{c}\left\langle T\left(\vec{r}_{c}\right)\right\rangle_{c}\langle g\rangle,(43) \\
& \eta_{\mathrm{cov}}^{2}=\frac{\int_{E} \int_{E^{\prime}} d E d E^{\prime} u\left(E-E_{\min }\right) u\left(E^{\prime}-E_{\min }\right)\left\langle\frac{w^{c}\left(E_{0}, E\right)}{\varepsilon_{\mathrm{e}-\mathrm{h}}(E)} S(E) \frac{w^{c}\left(E_{0}, E^{\prime}\right)}{\varepsilon_{\mathrm{e}-\mathrm{h}}\left(E^{\prime}\right)} S\left(E^{\prime}\right)\right\rangle_{c}\left\langle T^{2}\left(\vec{r}_{c}\right)\right\rangle_{c}}{\left(\int_{E} d E u\left(E-E_{\min }\right)\left\langle\frac{w^{c}\left(E_{0}, E\right)}{\varepsilon_{\mathrm{e}-\mathrm{h}}(E)} S(E)\right\rangle_{c}\right)^{2}\left\langle T\left(\vec{r}_{c}\right)\right\rangle_{c}^{2}},
\end{aligned}
$$




$$
\begin{aligned}
& \eta_{\mathrm{pair}}^{2}=\frac{\int_{E} d E u\left(E-E_{\min }\right)\left\langle F(E) \frac{w^{c}\left(E_{0}, E\right)}{\varepsilon_{\mathrm{e}-\mathrm{h}}(E)} S^{2}(E)\right\rangle_{c}\left\langle T^{2}\left(\vec{r}_{c}\right)\right\rangle_{c}}{\left(\int_{E} d E u\left(E-E_{\min }\right)\left\langle\frac{w^{c}\left(E_{0}, E\right)}{\varepsilon_{\mathrm{e}-\mathrm{h}}(E)} S(E)\right\rangle_{c}\right)^{2}\left\langle T\left(\vec{r}_{c}\right)\right\rangle_{c}^{2}}, \\
& \eta_{\mathrm{ph}}^{2}=\frac{1}{\int_{E} d E u\left(E-E_{\min }\right)\left\langle\frac{w^{c}\left(E_{0}, E\right)}{\varepsilon_{\mathrm{e}-\mathrm{h}}(E)} S(E) Q\right\rangle_{c}\left\langle T\left(\vec{r}_{c}\right)\right\rangle_{c}}-\frac{\int_{E} d E u\left(E-E_{\mathrm{min}}\right)\left\langle\frac{w^{c}\left(E_{0}, E\right)}{\varepsilon_{\mathrm{e}-\mathrm{h}}(E)} S^{2}(E)\right\rangle_{c}\left\langle T^{2}\left(\vec{r}_{c}\right)\right\rangle_{c}}{\left(\int_{E} d E u\left(E-E_{\min }\right)\left\langle\frac{w^{c}\left(E_{0}, E\right)}{\varepsilon_{\mathrm{e}-\mathrm{h}}(E)} S(E)\right\rangle_{c}\right)_{c}^{2}\left\langle T\left(\vec{r}_{c}\right)\right\rangle_{c}^{2}}, \\
& \eta_{\text {gain }}^{2}=\frac{1}{\int_{E} d E u\left(E-E_{\min }\right)\left\langle\frac{w^{c}\left(E_{0}, E\right)}{\varepsilon_{\mathrm{e}-\mathrm{h}}(E)} S(E) Q\right\rangle_{c}\left\langle T\left(\vec{r}_{c}\right)\right\rangle_{c}} \frac{\sigma_{g}^{2}}{\langle g\rangle^{2}}, \\
& \eta_{\text {noise }}^{2}=\frac{\sigma_{\text {noise }}^{2}}{\left\langle Q\left(E_{0}\right)\right\rangle^{2}} \text {. }
\end{aligned}
$$

As opposed to semiempirical formulae, the formulae (42)-(48) contain all information of dependence of all contributions to the energy resolution on scintillator characteristics and characteristics of the processes that occur in a scintillation detector at registration of incident particles.

For our consideration it is more convenient to introduce the differential light yield

$$
\frac{\partial Y^{c}\left(E_{0}, E\right)}{\partial E}=\frac{w^{c}\left(E_{0}, E\right)}{\varepsilon_{\mathrm{e}-\mathrm{h}}(E)} S(E) Q,
$$

and the total light yield from a scintillator for incident particles with the energy $E_{0}$

$$
Y^{c}\left(E_{0}\right)=\int_{E} d E u\left(E-E_{\min }\right) \frac{\partial Y^{c}\left(E_{0}, E\right)}{\partial E}=E_{0} L,
$$

where $L$ is the standard notation for specific light yield.

In this case, the average value and the relative variance are

$$
\begin{aligned}
& \left\langle Q\left(E_{0}\right)\right\rangle=\left\langle Y^{c}\left(E_{0}\right)\right\rangle_{c}\left\langle T\left(\vec{r}_{c}\right)\right\rangle_{c}\langle g\rangle,(51) \\
& \eta_{Q}^{2}=\eta_{\text {cov }}^{2}+\eta_{\text {pair }}^{2}+\eta_{\text {phe }}^{2}+\eta_{\text {gain }}^{2}+\eta_{\text {noise }}^{2},(52) \\
& \eta_{\text {cov }}^{2}=\frac{\int_{E} \int_{E^{\prime}} d E d E^{\prime} u\left(E-E_{\text {min }}\right) u\left(E^{\prime}-E_{\min }\right)\left\langle\frac{\partial Y^{c}\left(E_{0}, E\right)}{\partial E} \frac{\partial Y^{c}\left(E_{0}, E^{\prime}\right)}{\partial E^{\prime}}\right\rangle_{c}\left\langle T^{2}\left(\vec{r}_{c}\right)\right\rangle_{c}}{\left\langle Y^{c}\left(E_{0}\right)\right\rangle_{c}^{2}\left\langle T\left(\vec{r}_{c}\right)\right\rangle_{c}^{2}}-1,(53) \\
& \eta_{\text {pair }}^{2}=\frac{\int_{E} d E u\left(E-E_{\min }\right)\left\langle F(E) \frac{\partial Y^{c}\left(E_{0}, E\right)}{\partial E} S(E) Q\right\rangle_{c}\left\langle T^{2}\left(\vec{r}_{c}\right)\right\rangle_{c}}{\left.\left\langle E_{0}\right)\right\rangle_{c}^{2}\left\langle T\left(\vec{r}_{c}\right)\right\rangle_{c}^{2}},(54) \\
& \left.\eta_{\mathrm{ph}}^{2}=\frac{1}{\left\langle Y^{c}\left(E_{0}\right)\right\rangle_{c}\left\langle T\left(\vec{r}_{c}\right)\right\rangle_{c}}-\frac{\left.\partial Y^{c}\left(E_{0}\right)\right\rangle_{c}^{2}\left\langle T\left(\vec{r}_{c}\right)\right\rangle_{c}^{2}}{\left.\partial E_{0}, E\right)} S(E) Q\right\rangle_{c}\left\langle T^{2}\left(\vec{r}_{c}\right)\right\rangle_{c} \\
& \eta_{\text {gain }}^{2}=\frac{1}{\left\langle Y^{c}\left(E_{0}\right)\right\rangle_{c}\left\langle T\left(\vec{r}_{c}\right)\right\rangle_{c}} \frac{\sigma_{g}^{2}}{\langle g\rangle^{2}},(56)
\end{aligned}
$$




$$
\eta_{\text {noise }}^{2}=\frac{\sigma_{\text {noise }}^{2}}{\left\langle Q\left(E_{0}\right)\right\rangle^{2}}
$$

The average value and the relative variance become the most simple in the case when all incident particles interact with scintillator in the vicinity of the point $\vec{r}_{0}$, and the volume element in which the energy of incident particle converts to the light photons, is small enough that the coefficients $\left\langle T\left(\vec{r}_{c}\right)\right\rangle_{c}=T\left(\vec{r}_{0}\right)$ and $\left\langle T^{2}\left(\vec{r}_{c}\right)\right\rangle_{c}=T^{2}\left(\vec{r}_{0}\right)$ are almost constant, then

$$
\begin{aligned}
& \left\langle Q\left(E_{0}\right)\right\rangle=\left\langle Y^{c}\left(E_{0}\right)\right\rangle_{c} T\left(\vec{r}_{0}\right)\langle g\rangle,(58) \\
& \eta_{Q}^{2}=\eta_{Y}^{2}+\eta_{\text {pair }}^{2}+\eta_{\text {phe }}^{2}+\eta_{\text {gain }}^{2}+\eta_{\text {noise }}^{2},(59) \\
& \eta_{Y}^{2}=\frac{\iint_{E} d E d E^{\prime} u\left(E-E_{\text {min }}\right) u\left(E^{\prime}-E_{\text {min }}\right)\left\langle\frac{\partial Y^{c}\left(E_{0}, E\right)}{\partial E} \frac{\partial Y^{c}\left(E_{0}, E^{\prime}\right)}{\partial E^{\prime}}\right\rangle_{c}}{\left\langle Y^{c}\left(E_{0}\right)\right\rangle_{c}^{2}}-1,
\end{aligned}
$$

is the relative variance of the photodetector signal caused by the covariance of the differential light yield. This term is due to dependence of the differential light yield on the energy of secondary particles in the course of downconversion of the energy of an incident particle. If the differential light yield does not depend on the energy of the secondary particles, then the total light yield from a scintillator linearly depends on energy of an incident particle, and this term is equal to zero.

If we define the average value of the probability of the luminescent center activation, the average value of the Fano factor, and the average energy of the electron-hole pair production by expressions

$$
\begin{aligned}
& \left\langle Y^{c}\left(E_{0}\right)\right\rangle_{c}=\int_{E} d E u\left(E-E_{\min }\right)\left\langle\frac{\partial Y^{c}\left(E_{0}, E\right)}{\partial E}\right\rangle_{c}=\int_{E} d E u\left(E-E_{\min }\right)\left\langle\frac{w^{c}\left(E_{0}, E\right)}{\varepsilon_{\mathrm{e}-\mathrm{h}}(E)} S(E) Q\right\rangle_{c},(61) \\
& =\frac{\left\langle W^{c}\left(E_{0}\right)\right\rangle_{c}}{\left\langle\varepsilon_{\mathrm{e}-\mathrm{h}}\right\rangle}\langle S\rangle Q \cong \frac{E_{0}}{\left\langle\varepsilon_{\mathrm{e}-\mathrm{h}}\right\rangle}\langle S\rangle Q \\
& \int_{E} d E u\left(E-E_{\min }\right)\left\langle\frac{\partial Y^{c}\left(E_{0}, E\right)}{\partial E} S(E) Q\right\rangle_{c}=\int_{E} d E u\left(E-E_{\min }\right)\left\langle\frac{w^{c}\left(E_{0}, E\right)}{\varepsilon_{\mathrm{e}-\mathrm{h}}(E)} S^{2}(E) Q^{2}\right\rangle_{c}, \quad(62) \\
& =\frac{\left\langle W^{c}\left(E_{0}\right)\right\rangle_{c}}{\left\langle\varepsilon_{\mathrm{e}-\mathrm{h}}\right\rangle}\left\langle S^{2}\right\rangle Q^{2} \cong \frac{E_{0}}{\left\langle\varepsilon_{\mathrm{e}-\mathrm{h}}\right\rangle}\left\langle S^{2}\right\rangle Q^{2} \\
& \int_{E} d E u\left(E-E_{\min }\right)\left\langle F(E) \frac{\partial Y^{c}\left(E_{0}, E\right)}{\partial E} S(E) Q\right\rangle_{c}=\int_{E} d E u\left(E-E_{\min }\right)\left\langle F(E) \frac{w^{c}\left(E_{0}, E\right)}{\varepsilon_{\mathrm{e}-\mathrm{h}}(E)} S^{2}(E) Q^{2}\right\rangle_{c}, \\
& =\frac{\left\langle W^{c}\left(E_{0}\right)\right\rangle_{c}}{\left\langle\varepsilon_{\mathrm{e}-\mathrm{h}}\right\rangle}\langle F\rangle\left\langle S^{2}\right\rangle Q^{2} \cong \frac{E_{0}}{\left\langle\varepsilon_{\mathrm{e}-\mathrm{h}}\right\rangle}\langle F\rangle\left\langle S^{2}\right\rangle Q^{2}
\end{aligned}
$$

then the contributions of the last three terms to the relative variance, can be rewritten as follows

$$
\eta_{\text {pair }}^{2}+\eta_{p h}^{2}+\eta_{\text {gain }}^{2}+\eta_{\text {noise }}^{2}=\frac{\left\langle\varepsilon_{\mathrm{e}-\mathrm{h}}\right\rangle}{E_{0}}(\langle F\rangle-1) \frac{\left\langle S^{2}\right\rangle}{\langle S\rangle^{2}}+\frac{\left\langle\varepsilon_{\mathrm{e}-\mathrm{h}}\right\rangle}{E_{0}} \frac{1+\eta_{g}^{2}}{\langle S\rangle Q}+\frac{\sigma_{\text {noise }}^{2}}{\left\langle Q\left(E_{0}\right)\right\rangle^{2}},
$$

where $\eta_{g}^{2}=\sigma_{g}^{2} /\langle g\rangle^{2}$ is the relative variance of the gain.

\section{COVARIANCE BETWEEN SIGNALS OF PHOTODETECTORS OF A SCINTILLATION DETECTOR}

On the importance of the covariance between signals of photodetectors was emphasized in [9], [10]. From the PGF (9), the covariance between signals of photodetectors $Q_{n}(E)$ and $Q_{n^{\prime}}(E)$ of a scintillation spectrometer has the form 


$$
\begin{aligned}
& \operatorname{cov}\left(Q_{n}, Q_{n^{\prime}}\right)=\left.\frac{\partial^{2} f_{\left\{Q_{n}\right\}}\left[\left\{s_{n}\right\}\right]}{\partial s_{n} \partial s_{n^{\prime}}}\right|_{\left\{s_{n}=1\right\}}-\left.\left.\frac{\partial f_{\left\{Q_{n}\right\}}\left[\left\{s_{n}\right\}\right]}{\partial s_{n}}\right|_{\left\{s_{n}=1\right\}} \cdot \frac{\partial f_{\left\{Q_{n}\right\}}\left[\left\{s_{n}\right\}\right]}{\partial s_{n^{\prime}}}\right|_{\left\{s_{n}=1\right\}}= \\
& =\lim _{I J K \rightarrow \infty} \sum_{c} p_{\alpha i j k \alpha^{\prime} i^{\prime} j^{\prime} k^{\prime}}^{c} \sum_{\alpha=1}^{A} \sum_{i=1}^{I} \sum_{j=1}^{J} \sum_{k=1}^{K} N_{\alpha i j k}^{c}\left\langle v_{\alpha i j k}\right\rangle \chi_{n \alpha i j k}\left\langle g_{n}\right\rangle \sum_{\alpha^{\prime}=1}^{A} \sum_{i^{\prime}=1}^{I} \sum_{j^{\prime}=1}^{J} \sum_{k^{\prime}=1}^{K} N_{\alpha^{\prime} i^{\prime} k^{\prime} k^{\prime}}^{c}\left\langle v_{\alpha^{\prime} i^{\prime} j^{\prime} k^{\prime}}\right\rangle \chi_{n^{\prime} \alpha^{\prime} i^{\prime} j^{\prime} k^{\prime}}\left\langle g_{n^{\prime}}\right\rangle \\
& -\left(\lim _{I J K \rightarrow \infty} \sum_{c} p_{\alpha i j k}^{c} \sum_{\alpha=1}^{A} \sum_{i=1}^{I} \sum_{j=1}^{J} \sum_{k=1}^{K} N_{\alpha i j k}^{c}\left\langle v_{\alpha i j k}\right\rangle \chi_{n \alpha i j k}\left\langle g_{n}\right\rangle\right)\left(\lim _{I J K \rightarrow \infty} \sum_{c} p_{\alpha^{\prime} i^{\prime} j^{\prime} k^{\prime}}^{c} \sum_{\alpha^{\prime}=1}^{A} \sum_{i^{\prime}=1}^{I} \sum_{j^{\prime}=1}^{J} \sum_{k^{\prime}=1}^{K} N_{\alpha^{\prime} i^{\prime} j^{\prime} k^{\prime}}^{c}\left\langle v_{\alpha^{\prime} i^{\prime} j^{\prime} k^{\prime}}\right\rangle \chi_{n^{\prime} \alpha^{\prime} i^{\prime} j^{\prime} k^{\prime}}\left\langle g_{n^{\prime}}\right\rangle\right) \\
& +\lim _{I J K \rightarrow \infty} \sum_{c} p_{\alpha i j k}^{c} \sum_{\alpha=1}^{A} \sum_{i=1}^{I} \sum_{j=1}^{J} \sum_{k=1}^{K} N_{\alpha i j k}^{c} \chi_{n \alpha i j k}\left(\sigma_{v_{\alpha i j k}}^{2}-\left\langle v_{\alpha i j k}\right\rangle\right)\left\langle g_{n}\right\rangle \chi_{n^{\prime} \alpha i j k}\left\langle g_{n^{\prime}}\right\rangle
\end{aligned}
$$

(65)

As we move to the absorbed energy distribution in the detector volume, the covariance between signals of photodetectors turns to

$$
\begin{aligned}
& \operatorname{cov}\left(Q_{n}, Q_{n^{\prime}}\right)=\sum_{\alpha=1}^{A} \sum_{\alpha^{\prime}=1}^{A} \iint_{V} \int_{E} \int_{V^{\prime}} \int_{E^{\prime}} \int_{\Omega^{\prime}} d V d E d \vec{\Omega} d V^{\prime} d E^{\prime} d \vec{\Omega}^{\prime} u\left(E-E_{\alpha \min }(\vec{r}, E, \vec{\Omega})\right) u\left(E^{\prime}-E_{\alpha^{\prime} \min }\left(\vec{r}^{\prime}, E^{\prime}, \vec{\Omega}^{\prime}\right)\right) \times \\
& \left\langle\frac{w_{\alpha}^{c}\left(E_{0}, \vec{r}, E, \vec{\Omega}\right)}{\varepsilon_{\mathrm{e}-\mathrm{h} \alpha}(\vec{r}, E, \vec{\Omega})}\left\langle\chi_{n \alpha}(\vec{r}, E, \vec{\Omega})\right\rangle_{D}\left\langle g_{n}\right\rangle \frac{w_{\alpha^{\prime}}^{c}\left(E_{0}, \vec{r}^{\prime}, E^{\prime}, \vec{\Omega}^{\prime}\right)}{\varepsilon_{\mathrm{e}-\mathrm{h} \alpha^{\prime}}\left(\vec{r}^{\prime}, E^{\prime}, \vec{\Omega}^{\prime}\right)}\left\langle\chi_{n^{\prime} \alpha^{\prime}}\left(\vec{r}^{\prime}, E^{\prime}, \vec{\Omega}^{\prime}\right)\right\rangle_{D}\left\langle g_{n^{\prime}}\right\rangle\right\rangle_{c} \\
& -\sum_{\alpha=1}^{A} \iint_{V} \int_{E} d V d E d \vec{\Omega} u\left(E-E_{\alpha \min }(\vec{r}, E, \vec{\Omega})\right)\left\langle\frac{w_{\alpha}^{c}\left(E_{0}, \vec{r}, E, \vec{\Omega}\right)}{\varepsilon_{\mathrm{e}-\mathrm{h} \alpha}(\vec{r}, E, \vec{\Omega})}\left\langle\chi_{n \alpha}(\vec{r}, E, \vec{\Omega})\right\rangle_{D}\left\langle g_{n}\right\rangle\right\rangle_{c} \times \\
& \sum_{\alpha^{\prime}=1}^{A} \int_{V^{\prime}} \int_{E^{\prime}} \int_{\Omega^{\prime}} d V^{\prime} d E^{\prime} d \vec{\Omega}^{\prime} u\left(E^{\prime}-E_{\alpha^{\prime} \min }\left(\vec{r}^{\prime}, E^{\prime}, \vec{\Omega}^{\prime}\right)\right)\left\langle\frac{w_{\alpha^{\prime}}^{c}\left(E_{0}, \vec{r}^{\prime}, E^{\prime}, \vec{\Omega}^{\prime}\right)}{\varepsilon_{\mathrm{e}-\mathrm{h} \alpha^{\prime}}\left(\vec{r}^{\prime}, E^{\prime}, \vec{\Omega}^{\prime}\right)}\left\langle\chi_{n^{\prime} \alpha^{\prime}}\left(\vec{r}^{\prime}, E^{\prime}, \vec{\Omega}^{\prime}\right)\right\rangle_{D}\left\langle g_{n^{\prime}}\right\rangle\right\rangle_{c} \\
& +\sum_{\alpha=1}^{A} \int_{V} \int_{E} \int_{\Omega} d V d E d \vec{\Omega} u\left(E-E_{\alpha \min }(\vec{r}, E, \vec{\Omega})\right) \times \\
& \left\langle\frac{w_{\alpha}^{c}\left(E_{0}, \vec{r}, E, \vec{\Omega}\right)}{\varepsilon_{\mathrm{e}-\mathrm{h} \alpha}(\vec{r}, E, \vec{\Omega})}\left(F_{\alpha}(\vec{r}, E, \vec{\Omega})-1\right)\left\langle\chi_{n \alpha}(\vec{r}, E, \vec{\Omega})\right\rangle_{D}\left\langle g_{n}\right\rangle\left\langle\chi_{n^{\prime} \alpha}(\vec{r}, E, \vec{\Omega})\right\rangle_{D}\left\langle g_{n^{\prime}}\right\rangle\right\rangle_{c}
\end{aligned}
$$

The expressions (65) and (66) are the most general for the covariance between signals of photodetectors of a scintillation spectrometer.

If the secondary particles are only electrons, and the scintillator is uniform and isotropic with only one radiative transition mode, then for the local absorption of the energy of an incident particle the covariance between signals of photodetectors turns to $\operatorname{cov}\left(Q_{n}, Q_{n^{\prime}}\right)=\int_{E} \int_{E^{\prime}} d E d E^{\prime} u\left(E-E_{\min }\right) u\left(E^{\prime}-E_{\min }\right) \times$

$$
\begin{aligned}
& \left\langle\frac{w^{c}\left(E_{0}, E\right)}{\varepsilon_{\mathrm{e}-\mathrm{h}}(E)} S(E) Q \frac{w^{c}\left(E_{0}, E^{\prime}\right)}{\varepsilon_{\mathrm{e}-\mathrm{h}}\left(E^{\prime}\right)} S\left(E^{\prime}\right) Q\right\rangle_{c}\left\langle T_{n}\left(\vec{r}_{c}\right) T_{n^{\prime}}\left(\vec{r}_{c}\right)\right\rangle_{c}\left\langle g_{n}\right\rangle\left\langle g_{n^{\prime}}\right\rangle \\
& -\int_{E} d E u\left(E-E_{\min }\right)\left\langle\frac{w^{c}\left(E_{0}, E\right)}{\varepsilon_{\mathrm{e}-\mathrm{h}}(E)} S(E) Q\right\rangle_{c}\left\langle T_{n}\left(\vec{r}_{c}\right)\right\rangle_{c}\left\langle g_{n}\right\rangle \int_{E^{\prime}} d E^{\prime} u\left(E^{\prime}-E_{\min }\right)\left\langle\frac{w^{c}\left(E_{0}, E^{\prime}\right)}{\varepsilon_{\mathrm{e}-\mathrm{h}}\left(E^{\prime}\right)} S\left(E^{\prime}\right) Q\right\rangle_{c}\left\langle T_{n^{\prime}}\left(\vec{r}_{c}\right)\right\rangle_{c}\left\langle g_{n^{\prime}}\right\rangle \\
& +\int_{E} d E u\left(E-E_{\min }\right)\left\langle\frac{w^{c}\left(E_{0}, E\right)}{\varepsilon_{\mathrm{e}-\mathrm{h}}(E)}(F(E)-1) S^{2}(E) Q^{2}\right\rangle_{c}\left\langle T_{n}\left(\vec{r}_{c}\right) T_{n^{\prime}}\left(\vec{r}_{c}\right)\right\rangle_{c}\left\langle g_{n}\right\rangle\left\langle g_{n^{\prime}}\right\rangle
\end{aligned}
$$

(67)

The covariance between signals of photodetectors of a spectrometer underlies an experimental method of the Fano factor determination for the first time proposed in [11]. For determination of the Fano factor the use of the relative covariance between signals of photodetectors was proposed 


$$
\begin{aligned}
& \frac{\operatorname{cov}\left(Q_{n}, Q_{n^{\prime}}\right)}{\left\langle Q_{n}\right\rangle\left\langle Q_{n^{\prime}}\right\rangle}=\frac{\iint_{E} d E d E^{\prime} u\left(E-E_{\min }\right) u\left(E^{\prime}-E_{\min }\right)\left\langle\frac{w^{c}\left(E_{0}, E\right)}{\varepsilon_{\mathrm{e}-\mathrm{h}}(E)} S(E) \frac{w^{c}\left(E_{0}, E^{\prime}\right)}{\varepsilon_{\mathrm{e}-\mathrm{h}}\left(E^{\prime}\right)} S\left(E^{\prime}\right)\right\rangle_{c}}{\left.\left.\left\langle\int_{E} d E u\left(E-E_{\min }\right)\left\langle\frac{w^{c}\left(E_{0}, E\right)}{\varepsilon_{\mathrm{e}-\mathrm{h}}(E)} S(E)\right\rangle_{c}\right)^{2}\right) T_{n^{\prime}}\left(\vec{r}_{c}\right)\right\rangle_{c}}-1 \\
& +\frac{\int_{E} d E u\left(E-E_{\min }\right)\left\langle\frac{w^{c}\left(E_{0}, E\right)}{\varepsilon_{\mathrm{e}-\mathrm{h}}(E)}(F(E)-1) S^{2}(E)\right\rangle_{c}}{\left(\int_{E} d E u\left(E-E_{\min }\right)\left\langle\frac{w^{c}\left(E_{0}, E\right)}{\varepsilon_{\mathrm{e}-\mathrm{h}}(E)} S(E)\right\rangle_{c}\right)^{2}} \frac{\left\langle T_{n}\left(\vec{r}_{c}\right) T_{n^{\prime}}\left(\vec{r}_{c}\right)\right\rangle_{c}}{\left\langle T_{n}\left(\vec{r}_{c}\right)\right\rangle_{c}\left\langle T_{n^{\prime}}\left(\vec{r}_{c}\right)\right\rangle_{c}}
\end{aligned}
$$

(68)

It is necessary to emphasize that unlike a correlation coefficient

$$
\rho\left(Q_{n}, Q_{n^{\prime}}\right)=\frac{\operatorname{cov}\left(Q_{n}, Q_{n^{\prime}}\right)}{\sigma_{Q_{n}} \sigma_{Q_{n^{\prime}}}}
$$

which depends on all the variances of signals of the photodetectors, the relative covariance (68) does not depend on the gains and the noises of photodetectors electronics. It is the important advantage of the proposed experimental method, in comparison with existing methods of the Fano factor determination based on the subtraction of the electronic noise from the variance of the signal of the photodetector [12].

The expression (68) becomes simpler in the case if incident particles interact with a scintillator in the vicinity of the point $\vec{r}_{0}$, and the volume element in which the energy of incident particle converts to the light photons, is small enough that the coefficients $\left\langle T_{n}\left(\vec{r}_{0}\right)\right\rangle_{c}=T_{n}\left(\vec{r}_{0}\right)\left\langle T_{n^{\prime}}\left(\vec{r}_{0}\right)\right\rangle_{c}=T_{n^{\prime}}\left(\vec{r}_{0}\right)$ are almost constant. In this case $\left\langle T_{n}\left(\vec{r}_{0}\right) T_{n^{\prime}}\left(\vec{r}_{0}\right)\right\rangle_{c}=T_{n}\left(\vec{r}_{0}\right) T_{n^{\prime}}\left(\vec{r}_{0}\right)$ and the expression (68) reduces to

$$
\begin{aligned}
& \frac{\operatorname{cov}\left(Q_{n}, Q_{n^{\prime}}\right)}{\left\langle Q_{n}\right\rangle\left\langle Q_{n^{\prime}}\right\rangle}=\frac{\iint_{E} d E d E^{\prime} u\left(E-E_{\min }\right) u\left(E^{\prime}-E_{\min }\right)\left\langle\frac{w^{c}\left(E_{0}, E\right)}{\varepsilon_{\mathrm{e}-\mathrm{h}}(E)} S(E) \frac{w^{c}\left(E_{0}, E^{\prime}\right)}{\varepsilon_{\mathrm{e}-\mathrm{h}}\left(E^{\prime}\right)} S\left(E^{\prime}\right)\right\rangle_{c}}{\left(\int_{E} d E u\left(E-E_{\min }\right)\left\langle\frac{w^{c}\left(E_{0}, E\right)}{\varepsilon_{\mathrm{e}-\mathrm{h}}(E)} S(E)\right\rangle_{c}\right)^{2}}-1 \\
& +\frac{\int_{E} d E u\left(E-E_{\min }\right)\left\langle\frac{w^{c}\left(E_{0}, E\right)}{\varepsilon_{\mathrm{e}-\mathrm{h}}(E)}(F(E)-1) S^{2}(E)\right\rangle_{c}}{\left(\int_{E} d E u\left(E-E_{\min }\right)\left\langle\frac{w^{c}\left(E_{0}, E\right)}{\varepsilon_{\mathrm{e}-\mathrm{h}}(E)} S(E)\right\rangle_{c}\right)^{2}}
\end{aligned}
$$

As we move to the differential light yield and the total light yield

$$
\begin{aligned}
& \frac{\operatorname{cov}\left(Q_{n}, Q_{n^{\prime}}\right)}{\left\langle Q_{n}\right\rangle\left\langle Q_{n^{\prime}}\right\rangle}=\frac{\iint_{E} d E d E^{\prime} u\left(E-E_{\min }\right) u\left(E^{\prime}-E_{\min }\right)\left\langle\frac{\partial Y^{c}\left(E_{0}, E\right)}{\partial E} \frac{\partial Y^{c}\left(E_{0}, E^{\prime}\right)}{\partial E^{\prime}}\right\rangle_{c}}{\left\langle Y^{c}\left(E_{0}\right)\right\rangle_{c}^{2}}-1 \\
& +\frac{\int_{E} d E u\left(E-E_{\min }\right)\left\langle\frac{\partial Y^{c}\left(E_{0}, E\right)}{\partial E}(F(E)-1) S(E)\right\rangle_{c}}{\left\langle Y^{c}\left(E_{0}\right)\right\rangle_{c}^{2}},
\end{aligned}
$$

With expressions (61)-(63), the relative covariance between signals of photodetectors reduces to

$$
\frac{\operatorname{cov}\left(Q_{n}, Q_{n^{\prime}}\right)}{\left\langle Q_{n}\right\rangle\left\langle Q_{n^{\prime}}\right\rangle}=\eta_{Y}^{2}\left(E_{0}\right)+\frac{\left\langle\varepsilon_{\mathrm{e}-\mathrm{h}}\right\rangle}{E_{0}}(\langle F\rangle-1) \frac{\left\langle S^{2}\right\rangle}{\langle S\rangle^{2}} \text {. }
$$


The dependence of the last term on the inverse energy of incident particles allows separation of the contributions of the covariance in the light yield and the Fano factor, and its experimental determination from the dependence of the relative covariance on the energy of incident particles. As the contribution of the second term in (72) is inversely proportional to the number of electron-hole pairs, then for high energy of incident particles the relative covariance between signals of photodetectors defines the covariance of the differential light yield in the course of downconversion of the energy of an incident particle to energies of the secondary particles.

\section{CONCLUSION}

The exact mathematical description of process at registration of incident particles by a scintillation spectrometer with several photodetectors allows receiving correct formulas for the mean value and the variance of the amplitude at the output of photodetectors, and the covariance between signals of photodetectors. It was shown that using the relative covariance of signals allows the experimental determination of the covariance in the light yield and the Fano factor in a scintillator.

\section{REFERENCES}

[1] M. Moszyński, A. Syntfeld-Każuch, L. Swiderski, M. Grodzicka，J. Iwanowska，P. Sibczyński, T. Szczęśniak "Energy resolution of scintillation detectors", Nucl. Instr. Meth. in Phys. Res. A, vol. 805, pp. 25-35, 2016.

[2] P. Lecoq, A. Annenkov, A. Gektin, M. Korzhik, C. Pedrini, Inorganic Scintillators for Detector Systems, SpringerVerlag Berlin Heidelberg, 2006.

[3] Qi Li, J. Q. Grim, R. T. Williams, G. A. Bizarri, W. W. Moses "The role of hole mobility in scintillator proportionality", Nucl. Instr. Meth. in Phys. Res. A, vol. 652, pp. 288-291, 2011.

[4] W. Feller, An Introduction to Probability Theory and Its Applications, John Wiley and Sons, New York - London Sydney, 1968.

[5] V.V. Samedov, "Accounting for fluctuations of electronphoton showers in the theory of shower spectrometers", Ph.D. dissertation, Moscow Engineering Physics Institute, Moscow, 1972.

[6] V.V. Samedov, "Energy Resolution of Chernkov and Scintillation Shower Spectrometers for High-Energy Particles", Instruments and Experimental Techniques, vol. 28, pp. 1037-1039, 1985 .

[7] V.V. Samedov, "Correct method for calculating energy resolution and instrument functions of scintillation spectrometers", Measurement Techniques, vol. 28, pp. 265$271,1985$.

[8] U. Fano, "Ionization yield of radiation II, The fluctuation of the number of ions", Phys. Rev., vol. 72, pp. 26-29, 1947.

[9] V.V. Samedov, "Position resolution in DROIDs", J. Low Temp. Phys., vol. 151, pp. 333-336, 2008.

[10] V.V. Samedov, "Once More on the Position Resolution of DROIDs", AIP Conference Proceedings, vol. 1185, pp. 397-400, 2009.

[11] V.V. Samedov, "How to Determine Experimentally the Fano Factor in DROIDs", AIP Conference Proceedings, vol. 1185, pp. 462-464, 2009.

[12] R. Devanathan, L.R. Corrales, F. Gao, W.J. Weber, "Signal variance in gamma-ray detectors-A review", Nucl. Instr. Meth. in Phys. Res. A, vol. 565, pp. 637-649, 2006. 